\title{
A Kenmotsu metric as a conformal $\eta$-Einstein soliton
}

\author{
Roy S. ${ }^{1, \bowtie}$, Dey S. ${ }^{2}$, Bhattacharyya A. ${ }^{1}$
}

The object of the present paper is to study some properties of Kenmotsu manifold whose metric is conformal $\eta$-Einstein soliton. We have studied certain properties of Kenmotsu manifold admitting conformal $\eta$-Einstein soliton. We have also constructed a 3-dimensional Kenmotsu manifold satisfying conformal $\eta$-Einstein soliton.

Key words and phrases: Einstein soliton, $\eta$-Einstein soliton, conformal $\eta$-Einstein soliton, $\eta$-Einstein manifold, Kenmotsu manifold.

\footnotetext{
${ }^{1}$ Department of Mathematics, Jadavpur University, Kolkata, India

2 Department of Mathematics, Bidhan Chandra College, Asansol, West Bengal, India

$\bowtie$ Corresponding author

E-mail: soumendu1103mtma@gmail.com(Roy S.), santu.mathju@gmail.com (Dey S.), bhattachar1968@yahoo.co.in (Bhattacharyya A.)
}

\section{Introduction}

The notion of Einstein soliton was introduced by G. Catino and L. Mazzieri [3] in 2016, which generates self-similar solutions to Einstein flow

$$
\frac{\partial g}{\partial t}=-2\left(S-\frac{r}{2} g\right)
$$

where $S$ is Ricci tensor, $g$ is Riemannian metric and $r$ is the scalar curvature.

The equation of the $\eta$-Einstein soliton [2] is given by,

$$
£_{\xi} g+2 S+(2 \lambda-r) g+2 \mu \eta \otimes \eta=0
$$

where $£_{\xi}$ is the Lie derivative along the vector field $\xi, S$ is the Ricci tensor, $r$ is the scalar curvature of the Riemannian metric $g$, and $\lambda$ and $\mu$ are real constants. For $\mu=0$, the data $(g, \xi, \lambda)$ is called Einstein soliton.

In 2018, M.D. Siddiqi [6] introduced the notion of conformal $\eta$-Ricci soliton [8] as

$$
£_{\xi} g+2 S+\left[2 \lambda-\left(p+\frac{2}{n}\right)\right] g+2 \mu \eta \otimes \eta=0,
$$

where $£_{\xi}$ is the Lie derivative along the vector field $\xi, S$ is the Ricci tensor, $\lambda, \mu$ are constants, $p$ is a scalar non-dynamical field (time dependent scalar field) and $n$ is the dimension of manifold. For $\mu=0$, conformal $\eta$-Ricci soliton becomes conformal Ricci soliton [7].

$\mathrm{y} \triangle \mathrm{K} 514.76$

2020 Mathematics Subject Classification: 53C15, 53C25, 53C44.

The first author is supported by Swami Vivekananda Merit Cum Means Scholarship, Government of West Bengal, India. 
In [9], S. Roy, S. Dey and A. Bhattacharyya have defined conformal Einstein soliton, which can be written as

$$
£_{V} g+2 S+\left[2 \lambda-r+\left(p+\frac{2}{n}\right)\right] g=0
$$

where $\ell_{V}$ is the Lie derivative along the vector field $V, S$ is the Ricci tensor, $r$ is the scalar curvature of the Riemannian metric $g, \lambda$ is real constant, $p$ is a scalar non-dynamical field (time dependent scalar field) and $n$ is the dimension of manifold.

So we introduce the notion of conformal $\eta$-Einstein soliton as follows.

Definition 1. A Riemannian manifold $(M, g)$ of dimension $n$ is said to admit conformal $\eta$-Einstein soliton if

$$
£_{\xi} g+2 S+\left[2 \lambda-r+\left(p+\frac{2}{n}\right)\right] g+2 \mu \eta \otimes \eta=0,
$$

where $£_{\xi}$ is the Lie derivative along the vector field $\xi, \lambda, \mu$ are real contants and $S, r, p, n$ are same as defined in (1).

In the present paper, we study conformal $\eta$-Einstein soliton on Kenmotsu manifold. The paper is organized as follows.

After introduction, section 2 is devoted for preliminaries on $(2 n+1)$-dimensional Kenmotsu manifold. In section 3, we have studied conformal $\eta$-Einstein soliton on Kenmotsu manifold. Here we proved that if a $(2 n+1)$-dimensional Kenmotsu manifold admits conformal $\eta$-Einstein soliton then the manifold becomes $\eta$-Einstein. We have also characterized the nature of the manifold if the manifold is Ricci symmetric and the Ricci tensor is $\eta$-recurrent. Also we have discussed the condition, when the manifold has cyclic Ricci tensor. Then we have obtained the conditons in a $(2 n+1)$-dimensional Kenmotsu manifold admitting conformal $\eta$-Einstein soliton, when a vector field $V$ is pointwise co-linear with $\xi$ and a $(0,2)$-tensor field $h$ is parallel with respect to the Levi-Civita connection associated to $g$. We have also examined the nature of a Ricci-recurrent Kenmotsu manifold admitting conformal $\eta$-Einstein soliton.

In last section, we have given an example of a 3-dimensional Kenmotsu manifold satisfying conformal $\eta$-Einstein soliton.

\section{Preliminaries}

Let $M$ be a $(2 n+1)$-dimensional connected almost contact metric manifold with an almost contact metric structure $(\phi, \xi, \eta, g)$, where $\phi$ is a $(1,1)$-tensor field, $\xi$ is a vector field, $\eta$ is a 1 -form and $g$ is the compatible Riemannian metric such that

$$
\begin{gathered}
\phi^{2}(X)=-X+\eta(X) \xi, \eta(\xi)=1, \eta \circ \phi=0, \phi \xi=0, \\
g(\phi X, \phi Y)=g(X, Y)-\eta(X) \eta(Y), \\
g(X, \phi Y)=-g(\phi X, Y), \\
g(X, \xi)=\eta(X),
\end{gathered}
$$

for all vector fields $X, Y \in \chi(M)$.

The fundamental 2-form $\Phi$ on an almost contact metric manifold $M^{2 n+1}$ is defined by $\Phi(X, Y)=g(X, \phi Y)$ for any vector fields $X$ and $Y$ on $M^{2 n+1}$. In an almost contact metric manifold, we have $\eta \wedge \Phi^{n} \neq 0$. 
When $\Phi=d \eta$, an almost contact metric manifold becomes contact metric manifold.

An almost contact metric manifold satisfying $d \eta=0$ and $d \Phi=2 \eta \wedge \Phi$ is said to be an almost Kenmotsu manifold [4].

An almost contact metric manifold is said to be a Kenmotsu manifold [5] if

$$
\begin{gathered}
\left(\nabla_{X} \phi\right) Y=-g(X, \phi Y) \xi-\eta(Y) \phi X \\
\nabla_{X} \xi=X-\eta(X) \xi
\end{gathered}
$$

where $\nabla$ denotes the Riemannian connection of $g$.

In a Kenmotsu manifold the following relations hold [1]:

$$
\begin{gathered}
\eta(R(X, Y) Z)=g(X, Z) \eta(Y)-g(Y, Z) \eta(X), \\
R(X, Y) \xi=\eta(X) Y-\eta(Y) X, \\
R(X, \xi) Y=g(X, Y) \xi-\eta(Y) X,
\end{gathered}
$$

where $R$ is the Riemannian curvature tensor,

$$
\begin{gathered}
S(X, \xi)=-2 n \eta(X) \\
S(\phi X, \phi Y)=S(X, Y)+2 n \eta(X) \eta(Y), \\
\left(\nabla_{X} \eta\right) Y=g(X, Y)-\eta(X) \eta(Y)
\end{gathered}
$$

for all vector fields $X, Y, Z \in \chi(M)$.

Now we know,

$$
\left(£_{\xi} g\right)(X, Y)=g\left(\nabla_{X} \xi, Y\right)+g\left(X, \nabla_{Y} \xi\right),
$$

for all vector fields $X, Y, \in \chi(M)$. Then using (6) and (10), we get,

$$
\left(£_{\xi} g\right)(X, Y)=2[g(X, Y)-\eta(X) \eta(Y)] .
$$

\section{Conformal $\eta$-Einstein soliton on Kenmotsu manifold}

Let $M$ be a $(2 n+1)$-dimensional Kenmotsu manifold. Consider the conformal $\eta$-Einstein soliton (2) on $M$ as

$$
\left(£_{\xi} g\right)(X, Y)+2 S(X, Y)+\left[2 \lambda-r+\left(p+\frac{2}{2 n+1}\right)\right] g(X, Y)+2 \mu \eta(X) \eta(Y)=0
$$

for all vector fields $X, Y, \in \chi(M)$.

Theorem 1. If the metric of a $(2 n+1)$-dimensional Kenmotsu manifold is a conformal $\eta$-Einstein soliton, then the manifold becomes $\eta$-Einstein and the scalar curvature is

$$
\left(p+\frac{2}{2 n+1}\right)-4 n+2 \lambda+2 \mu
$$


Proof. First, using (11), the above equation becomes,

$$
S(X, Y)=-\left[\lambda-\frac{r}{2}+\frac{\left(p+\frac{2}{2 n+1}\right)}{2}+1\right] g(X, Y)-(\mu-1) \eta(X) \eta(Y) .
$$

Taking $Y=\xi$ in the above equation and using (8), we get,

$$
r=\left(p+\frac{2}{2 n+1}\right)-4 n+2 \lambda+2 \mu
$$

since $\eta(X) \neq 0$, for all $X \in \chi(M)$.

Also from (12), it follows that the manifold is $\eta$-Einstein and this completes the proof.

Theorem 2. If the metric of a $(2 n+1)$-dimensional Ricci symmteric Kenmotsu manifold is a conformal $\eta$-Einstein soliton, then $\mu=1$ and the scalar curvature is $\left(p+\frac{2}{2 n+1}\right)-4 n+2 \lambda+2$.

Proof. We know, that $\left(\nabla_{X} S\right)(Y, Z)=X(S(Y, Z))-S\left(\nabla_{X} Y, Z\right)-S\left(Y, \nabla_{X} Z\right)$, for all vector fields $X, Y, Z$ on $M$ and $\nabla$ is the Levi-Civita connection associated with $g$.

Now replacing the expression of $S$ from (12), we obtain

$$
\left(\nabla_{X} S\right)(Y, Z)=-(\mu-1)\left[\eta(Z)\left(\nabla_{X} \eta\right) Y+\eta(Y)\left(\nabla_{X} \eta\right) Z\right]
$$

for all vector fields $X, Y, Z$ on $M$.

As the manifold $M$ is Ricci symmetric, i.e $\nabla S=0$.

Then from (14), we get

$$
-(\mu-1)\left[\eta(Z)\left(\nabla_{X} \eta\right) Y+\eta(Y)\left(\nabla_{X} \eta\right) Z\right]=0
$$

for all vector fields $X, Y, Z \in \chi(M)$.

Taking $Z=\xi$ in the above equation and using (9), (3), we obtain, $\mu=1$.

Then from (13), we get

$$
r=\left(p+\frac{2}{2 n+1}\right)-4 n+2 \lambda+2
$$

Hence, we complete the proof.

Theorem 3. If the metric of a $(2 n+1)$-dimensional Kenmotsu manifold is a conformal $\eta$-Einstein soliton and the Ricci tensor $S$ is $\eta$-recurrent, then the scalar curvature is

$$
2 \lambda+2 \mu+\left(p+\frac{2}{2 n+1}\right)
$$

Proof. If the Ricci tensor $S$ is $\eta$-recurrent, then we have $\nabla S=\eta \otimes S$, which implies

$$
\left(\nabla_{X} S\right)(Y, Z)=\eta(X) S(Y, Z)
$$

for all vector fields $X, Y, Z$ on $M$. Using (14), the above equation reduces to

$$
-(\mu-1)\left[\eta(Z)\left(\nabla_{X} \eta\right) Y+\eta(Y)\left(\nabla_{X} \eta\right) Z\right]=\eta(X) S(Y, Z) .
$$

Taking $Y=\xi, Z=\xi$ in the above equation and using (9), (12), we get

$$
\left[\lambda+\mu-\frac{r}{2}+\frac{p+\frac{2}{2 n+1}}{2}\right] \eta(X)=0,
$$

which implies

$$
r=2 \lambda+2 \mu+\left(p+\frac{2}{2 n+1}\right)
$$

This completes the proof. 
Theorem 4. Let the metric of a $(2 n+1)$-dimensional Kenmotsu manifold $M$ is a conformal $\eta$-Einstein soliton. Then $M$ has cyclic Ricci tensor if $\mu=1$.

Proof. Similarly from (14), we get

$$
\left(\nabla_{Y} S\right)(Z, X)=-(\mu-1)\left[\eta(X)\left(\nabla_{Y} \eta\right) Z+\eta(Z)\left(\nabla_{X} \eta\right) Y\right]
$$

and

$$
\left(\nabla_{Z} S\right)(X, Y)=-(\mu-1)\left[\eta(Y)\left(\nabla_{Z} \eta\right) X+\eta(X)\left(\nabla_{Z} \eta\right) Y\right]
$$

for all vector fields $X, Y, Z$ on $M$.

Then adding (14), (15), (16) and using (9), (4), we obtain

$$
\begin{aligned}
\left(\nabla_{X} S\right)(Y, Z)+\left(\nabla_{Y} S\right)(Z, X)+\left(\nabla_{Z} S\right)(X, Y)= & -2(\mu-1)[\eta(X) g(\phi Y, \phi Z) \\
& +\eta(Y) g(\phi Z, \phi X)+\eta(Z) g(\phi X, \phi Y)] .
\end{aligned}
$$

Now, as the manifold $M$ has cyclic Ricci tensor, i.e

$$
\left(\nabla_{X} S\right)(Y, Z)+\left(\nabla_{Y} S\right)(Z, X)+\left(\nabla_{Z} S\right)(X, Y)=0
$$

then from (17), we have

$$
(\mu-1)[\eta(X) g(\phi Y, \phi Z)+\eta(Y) g(\phi Z, \phi X)+\eta(Z) g(\phi X, \phi Y)]=0 .
$$

Taking $X=\xi$ in the above equation and using (3), we get $\mu=1$.

Again, if we take $\mu=1$ in (17), we obtaion $\left(\nabla_{X} S\right)(Y, Z)+\left(\nabla_{Y} S\right)(Z, X)+\left(\nabla_{Z} S\right)(X, Y)=0$, i.e the manifold $M$ has cyclic Ricci tensor and this completes the proof.

Corollary 1. If a $(2 n+1)$-dimensional Kenmotsu manifold $M$ has a cyclic Ricci tensor and the metric is a conformal $\eta$-Einstein soliton, then the scalar curvature is $\left(p+\frac{2}{2 n+1}\right)-4 n+2 \lambda+2$.

Proof. If $\mu=1$, then from (13) we obtain $r=\left(p+\frac{2}{2 n+1}\right)-4 n+2 \lambda+2$.

Theorem 5. Let $M$ be a $(2 n+1)$-dimensional Kenmotsu manifold admitting a conformal $\eta$-Einstein soliton $(g, V), V$ being a vector field on $M$. If $V$ is pointwise co-linear with $\xi$, a vector field on $M$, then $V$ is a constant multiple of $\xi$, provided the scalar curvature is

$$
2 \lambda+2 \mu+\left(p+\frac{2}{2 n+1}\right)-4 n
$$

Proof. A conformal $\eta$-Einstein soliton is defined on a $(2 n+1)$-dimensional Kenmotsu manifold $M$ as

$$
£_{V} g+2 S+\left[2 \lambda-r+\left(p+\frac{2}{2 n+1}\right)\right] g+2 \mu \eta \otimes \eta=0
$$

where $£_{V}$ is the Lie derivative along the vector field $V, S$ is the Ricci tensor, $r$ is the scalar curvature of the Riemannian metric $g, \lambda, \mu$ are real contants, $p$ is a scalar non-dynamical field (time dependent scalar field).

Since, $V$ is pointwise co-linear with $\xi$, let $V=b \xi$, where $b$ is a function on $M$.

Then (18) becomes

$$
\left(£_{b \xi} g\right)(X, Y)+2 S(X, Y)+\left[2 \lambda-r+\left(p+\frac{2}{2 n+1}\right)\right] g(X, Y)+2 \mu \eta(X) \eta(Y)=0
$$


for all vector fields $X, Y$ on $M$. Applying the property of Lie derivative and Levi-Civita connection, we have

$$
\begin{aligned}
b g\left(\nabla_{X} \xi, Y\right)+(X b) \eta(Y) & +b g\left(\nabla_{Y} \xi, X\right)+(Y b) \eta(X)+2 S(X, Y) \\
& +\left[2 \lambda-r+\left(p+\frac{2}{2 n+1}\right)\right] g(X, Y)+2 \mu \eta(X) \eta(Y)=0 .
\end{aligned}
$$

Now using (6), we get

$$
\begin{aligned}
2 b g(X, Y)-2 b \eta(X) \eta(Y) & +(X b) \eta(Y)+(Y b) \eta(X)+2 S(X, Y) \\
& +\left[2 \lambda-r+\left(p+\frac{2}{2 n+1}\right)\right] g(X, Y)+2 \mu \eta(X) \eta(Y)=0
\end{aligned}
$$

Taking $Y=\xi$ in the above equation and using (3), (5), (8), we obtain

$$
(X b)+(\xi b) \eta(X)-4 n \eta(X)+\left[2 \lambda-r+\left(p+\frac{2}{2 n+1}\right)\right] \eta(X)+2 \mu \eta(X)=0 .
$$

Then by putting $X=\xi$, the above equation reduces to

$$
\xi b=2 n+\frac{r}{2}-\lambda-\mu-\frac{\left(p+\frac{2}{2 n+1}\right)}{2} .
$$

Using (20), equation (19) becomes

$$
(X b)+\left[\lambda+\mu+\frac{\left(p+\frac{2}{2 n+1}\right)}{2}-2 n-\frac{r}{2}\right] \eta(X)=0 .
$$

Applying exterior differentiation in (21), we have

$$
\left[\lambda+\mu+\frac{\left(p+\frac{2}{2 n+1}\right)}{2}-2 n-\frac{r}{2}\right] d \eta=0
$$

Now we know

$$
d \eta(X, Y)=\frac{1}{2}\left[\left(\nabla_{X} \eta\right) Y-\left(\nabla_{Y} \eta\right) X\right]
$$

for all vector fields $X, Y$ on $M$. Using (9), the above equation becomes $d \eta=0$. Hence the 1-form $\eta$ is closed.

So from (22), either $r=2 \lambda+2 \mu+\left(p+\frac{2}{2 n+1}\right)-4 n$ or $r \neq 2 \lambda+2 \mu+\left(p+\frac{2}{2 n+1}\right)-4 n$. If $r=2 \lambda+2 \mu+\left(p+\frac{2}{2 n+1}\right)-4 n,(21)$ reduces to $(X b)=0$. This implies that $b$ is constant and this completes the proof.

Theorem 6. In a $(2 n+1)$-dimensional Kenmotsu manifold assume that a symmetric $(0,2)$-tensor field $h=£_{\xi} g+2 S+2 \mu \eta \otimes \eta$ is parallel with respect to the Levi-Civita connection associated to $g$. Then $(g, \xi)$ yields a conformal $\eta$-Einstein soliton.

Proof. Note that $h$ is a symmetric tensor field of $(0,2)$-type, which we suppose to be parallel with respect to the Levi-Civita connection $\nabla$, i.e $\nabla h=0$. Applying the Ricci commutation identity, we have

$$
\nabla^{2} h(X, Y ; Z, W)-\nabla^{2} h(X, Y ; W, Z)=0
$$


for all vector fields $X, Y, Z, W$ on $M$. From the above equation we obtain the relation

$$
h(R(X, Y) Z, W)+h(Z, R(X, Y) W)=0 .
$$

Replacing $Z=W=\xi$ in the above equation and using (7), we get

$$
\eta(X) h(Y, \xi)-\eta(Y) h(X, \xi)=0
$$

Replacing $X=\xi$ and using (3), the above equation reduces to

$$
h(Y, \xi)=\eta(Y) h(\xi, \xi)
$$

for all vector fields $Y$ on $M$. Differentiating the above equation covariantly with respect to $X$, we get

$$
\nabla_{X}(h(Y, \xi))=\nabla_{X}(\eta(Y) h(\xi, \xi)) .
$$

Now expanding the above eqution by using (23), (6), (9) and the property $\nabla h=0$, we obtain

$$
h(X, Y)=h(\xi, \xi) g(X, Y)
$$

for all vector fields $X, Y$ on $M$.

Let us take

$$
h=£_{\xi} g+2 S+2 \mu \eta \otimes \eta .
$$

Then from (11), (12), we get

$$
h(\xi, \xi)=-2 \lambda-\left(p+\frac{2}{2 n+1}\right)+r .
$$

Then using (25), equation (24) becomes

$$
\left(£_{\xi} g\right)(X, Y)+2 S(X, Y)+\left[2 \lambda-r+\left(p+\frac{2}{2 n+1}\right)\right] g(X, Y)+2 \mu \eta(X) \eta(Y)=0,
$$

which is the conformal $\eta$-Einstein soliton. Hence, we complete the proof.

Definition 2. A Kenmotsu manifold is said to be Ricci-recurrent manifold if there exists a nonzero 1-form $A$ such that

$$
\left(\nabla_{W} S\right)(Y, Z)=A(W) S(Y, Z)
$$

for any vector fields $W, Y, Z$ on $M$.

Theorem 7. If the metric of a $(2 n+1)$-dimensional Ricci-recurrent Kenmotsu manifold is a conformal $\eta$-Einstein soliton with the 1-form $A$, then the scalar curvature becomes

$$
2 \lambda+2 \mu+\left(p+\frac{2}{2 n+1}\right)+4 n(A(\xi)-1) .
$$

Proof. Replacing $Z$ by $\xi$ in the equation (26) and using (8), we get

$$
\left(\nabla_{W} S\right)(Y, \xi)=-2 n A(W) \eta(Y)
$$

which implies that

$$
W S(Y, \xi)-S\left(\nabla_{W} Y, \xi\right)-S\left(Y, \nabla_{W} \xi\right)=-2 n A(W) \eta(Y) .
$$


Using (8) and (6), the above equation becomes

$$
2 n\left(\nabla_{W} \eta\right) Y+2 n \eta(W) \eta(Y)+S(Y, W)=2 n A(W) \eta(Y)
$$

Again using (9), the above equation reduces to

$$
2 n g(W, Y)+S(Y, W)=2 n A(W) \eta(Y) .
$$

Taking $W=\xi$ in the above equation and using (12), we obtain

$$
r=2 \lambda+2 \mu+\left(p+\frac{2}{2 n+1}\right)+4 n(A(\xi)-1) .
$$

This completes the proof.

Example 1. Here, we consider the three-dimensional manifold $M=\left\{(x, y, z) \in \mathbb{R}^{3},(x, y, z) \neq\right.$ $(0,0,0)\}$, where $(x, y, z)$ are standard coordinates in $\mathbb{R}^{3}$. The vector fields

$$
e_{1}=z \frac{\partial}{\partial x}, \quad e_{2}=z \frac{\partial}{\partial y}, \quad e_{3}=-z \frac{\partial}{\partial z}
$$

are linearly independent at each point of $M$. Let $g$ be the Riemannian metric defined by

$$
g\left(e_{1}, e_{2}\right)=g\left(e_{2}, e_{3}\right)=g\left(e_{3}, e_{1}\right)=0, \quad g\left(e_{1}, e_{1}\right)=g\left(e_{2}, e_{2}\right)=g\left(e_{3}, e_{3}\right)=1 .
$$

Let $\eta$ be the 1-form defined by $\eta(Z)=g\left(Z, e_{3}\right)$ for any vector field $Z$ in $\mathbb{R}^{3}$ and $\phi$ be the $(1,1)$-tensor field defined by $\phi e_{1}=-e_{2}, \phi e_{2}=e_{1}, \phi e_{3}=0$. Then using the linearity of $\phi$ and $g$, we have

$$
\eta\left(e_{3}\right)=1, \quad \phi^{2} Z=-Z+\eta(Z) e_{3}, \quad g(\phi Z, \phi W)=g(Z, W)-\eta(Z) \eta(W)
$$

for any $Z, W \in \chi(M)$. Thus for $e_{3}=\xi,(\phi, \xi, \eta, g)$ defines an almost contact metric structure on $M$.

Let $\nabla$ be the Levi-Civita connection with respect to the Riemannian metric $g$. Then we have $\left[e_{1}, e_{2}\right]=0,\left[e_{1}, e_{3}\right]=e_{1},\left[e_{2}, e_{3}\right]=e_{2}$. The connection $\nabla$ of the metric $g$ is given by

$$
2 g\left(\nabla_{X} Y, Z\right)=X g(Y, Z)+Y g(Z, X)-Z g(X, Y)-g(X,[Y, Z])-g(Y,[X, Z])+g(Z,[X, Y])
$$

which is known as Koszul's formula.

Using Koszul's formula, we can easily calculate

$$
\begin{array}{lll}
\nabla_{e_{1}} e_{1}=-e_{3}, & \nabla_{e_{1}} e_{2}=0, & \nabla_{e_{1}} e_{3}=e_{1}, \\
\nabla_{e_{2}} e_{1}=0, & \nabla_{e_{2}} e_{2}=-e_{3}, & \nabla_{e_{2}} e_{3}=e_{2}, \\
\nabla_{e_{3}} e_{1}=0, & \nabla_{e_{3}} e_{2}=0, & \nabla_{e_{3}} e_{3}=0 .
\end{array}
$$

From the above it follows that the manifold satisfies $\nabla_{X} \xi=X-\eta(X) \xi$ for $\xi=e_{3}$. Hence the manifold is a Kenmotsu manifold. So, here we have considered $\mathbb{R}^{3}$ as an almost contact manifold, which turns out to be a 3-dimensional Kenmotsu manifold.

Also, the Riemannian curvature tensor $R$ is given by

$$
R(X, Y) Z=\nabla_{X} \nabla_{Y} Z-\nabla_{Y} \nabla_{X} Z-\nabla_{[X, Y]} Z
$$


Hence,

$$
\begin{array}{lll}
R\left(e_{1}, e_{2}\right) e_{2}=-e_{1}, & R\left(e_{1}, e_{3}\right) e_{3}=-e_{1}, & R\left(e_{2}, e_{1}\right) e_{1}=-e_{2}, \\
R\left(e_{2}, e_{3}\right) e_{3}=-e_{2}, & R\left(e_{3}, e_{1}\right) e_{1}=-e_{3}, & R\left(e_{3}, e_{2}\right) e_{2}=-e_{3}, \\
R\left(e_{1}, e_{2}\right) e_{3}=0, & R\left(e_{2}, e_{3}\right) e_{1}=0, & R\left(e_{3}, e_{1}\right) e_{2}=0 .
\end{array}
$$

Then, the Ricci tensor $S$ is given by $S\left(e_{1}, e_{1}\right)=-2, S\left(e_{2}, e_{2}\right)=-2, S\left(e_{3}, e_{3}\right)=-2$. From (12), we have

$$
S\left(e_{3}, e_{3}\right)=-\left[\lambda+\mu-\frac{r}{2}+\frac{\left(p+\frac{2}{3}\right)}{2}\right],
$$

which implies that

$$
r=2 \lambda+2 \mu-4+\left(p+\frac{2}{3}\right) .
$$

Hence $\lambda$ and $\mu$ satisfies equation (13) and so $g$ defines a conformal $\eta$-Einstein soliton on the 3-dimensional Kenmotsu manifold $M$.

\section{References}

[1] Bagewadi C.S., Prasad V.S. Notes on Kenmotsu manifolds. Bull. Calcutta Math. Soc. 1999, 91 (5), 379-384.

[2] Blaga A.M. On Gradient $\eta$-Einstein Solitons. Kragujevac J. Math. 2018, 42 (2), 229-237. doi:10.5937/KgJMath1802229B

[3] Catino G., Mazzieri L. Gradient Einstein solitons. Nonlinear Anal. 2016, 132, 66-94. doi:10.1016/j.na.2015.10.021

[4] Janssens D., Vanhecke L. Almost contact structures and curvature tensors. Kodai Math. J. 1981, 4 (1), 1-27. doi: $10.2996 / \mathrm{kmj} / 1138036310$

[5] Kenmotsu K. A class of almost contact Riemannian manifolds. Tohoku Math. J. (2) 1972, 24 (1), 93-103. doi:10.2748/tmj/1178241594

[6] Siddiqi M.D. Conformal $\eta$-Ricci solitons in $\delta$-Lorentzian Trans Sasakian manifolds. Intern. J. Maps in Math. 2018, $1(1), 15-34$.

[7] Roy S., Bhattacharyya A. Conformal Ricci solitons on 3-dimensional trans-Sasakian manifold. Jordan J. Math. Stat. 2020, 13 (1), 89-109.

[8] Roy S., Dey S., Bhattacharyya A., Hui S.K. *-Conformal $\eta$-Ricci Soliton on Sasakian manifold. Asian-Eur. J. Math. doi: 10.1142/S1793557122500358

[9] Roy S., Dey S., Bhattacharyya A. Conformal Einstein soliton within the framework of para-Kähler manifold. arXiv:2005.05616v1 [math.DG].

Received 22.04.2020

Revised 01.08.2020

Рой С., Аей С., Бхаттачарія А. Метрика Кенмоцу як конформний ү-солітон Айнштайна // Карпатські матем. публ. - 2021. - Т.13, №1. - С. 110-118.

Метою даної роботи є вивчення деяких властивостей многовида Кенмоцу, метрика якого $€$ конформним $\eta$-солітоном Айнштайна. Ми дослідили певні властивості многовида Кенмоцу, що допускає конформний $\eta$-солітон Айнштайна. Також ми збудували тривимірний многовид Кенмоцу, шо задовольняє конформний $\eta$-солітон Айнштайна.

Ключові слова і фрази: солітон Айнштайна, $\eta$-солітон Айнштайна, конформний $\eta$-солітон Айнштайна, $\eta$-многовид Айнштайна, многовид Кенмоцу. 